\author{
Jesús Page $\cdot$ Roberto de la Fuente $\cdot$ Rocío Gómez • \\ Adela Calvente $\cdot$ Alberto Viera $\cdot$ María Teresa Parra \\ Juan Luis Santos · Soledad Berríos • \\ Raúl Fernández-Donoso • José Ángel Suja • \\ Julio S. Rufas
}

\title{
Sex chromosomes, synapsis, and cohesins: a complex affair
}

\begin{abstract}
During first meiotic prophase, homologous chromosomes are held together by the synaptonemal complex, a tripartite proteinaceous structure that extends along the entire length of meiotic bivalents. While this feature is applicable for autosomes, sex chromosomes often escape from this rule. Many species present sex chromosomes that differ between them in their morphology, length, and gene content. Moreover, in some species, sex chromosomes appear in a single dose in one of the sexes. In all of these cases, the behavior of sex chromosomes during meiosis is conspicuously affected, and this includes the assembly and dynamics of the synaptonemal complex. We review in this study the structure of the synaptonemal complex in the sex chromosomes of three groups of organisms, namely: mammals, orthopterans, and hemipterans, which present different patterns of sex chromosome structure and behavior. Of special interest is the analysis of
\end{abstract}

J. Page $(\bowtie) \cdot$ R. de la Fuente · R. Gómez · A. Calvente ·

A. Viera · M. T. Parra · J. Á. Suja · J. S. Rufas

Departamento de Biología, Edificio de Ciencias Biológicas,

Universidad Autónoma de Madrid, Cantoblanco,

Madrid 28049, Spain

e-mail: jesus.page@uam.es

Tel.: +34-914978242

Fax: +34-914978344

J. L. Santos

Departamento de Genética, Facultad de Biología,

Universidad Complutense,

Madrid, Spain

S. Berríos $\cdot$ R. Fernández-Donoso

Programa de Genética Humana,

Instituto de Ciencias Biomédicas,

Facultad de Medicina,

Universidad de Chile,

Santiago, Chile the organization of the axial/lateral elements of the synaptonemal complex in relation to other axial structures organized along meiotic chromosomes, mainly the cohesin axis. The differences found in the behavior of both axial structures reveal that while the organization of a cohesin axis along sex chromosomes is a conserved feature in most organisms and it shows very little morphological variations, the axial/lateral elements of the synaptonemal complex present a wide range of structural modifications on these chromosomes.

\section{Introduction}

The formation of the synaptonemal complex (SC) is one of the most relevant features of the first meiotic prophase. During leptotene, the components that constitute the axial elements (AEs) start to organize along chromosomes. The AEs of homologous chromosomes, converted into lateral elements (LEs) when synapsis begins during zygotene, are held together by the association of transverse filaments (TFs). Thus, the mature (tripartite) SC, which during the pachytene stage extends all along the bivalents, is composed by two LEs (one per homologue) and the central element (CE) that results from the overlapping of TFs in the central region of the SC (Moses 1956; Fawcett 1956).

It has been proposed that other axial structures organized along meiotic chromosomes may have an interaction with the elements of the SC. One of these structures is the chromosome scaffold, a structural framework that organizes along mitotic chromosomes (Paulson and Laemmli 1977) and whose main components are DNA topoisomerase II $\alpha$ and the condensin subunit SMC2 (Earnshaw et al. 1985; Saitoh et al. 1994). Because DNA topoisomerase II $\alpha$ is associated to the LEs during meiosis, it has been proposed that the scaffold and the SC may have some degree of interaction (Moens and Earnshaw 1989). This proposal is reinforced by the structural relationship found between the SC and the chromatid cores of meiotic bivalents (Rufas et al. 1992). More recently, an axis composed by cohesin proteins has been described associated to meiotic 
chromosomes (Pelttari et al. 2001). This structure has a location very similar to the LEs and appears all along the meiotic bivalents during the first meiotic prophase. It is interesting to note that the cohesin axis is organized even in the absence of the LEs, and is able to recruit the components of the TFs to form a central element-like structure (Pelttari et al. 2001). This indicates that the LEs and the cohesin axes are distinct and independent structures.

Although SC function(s) still remains controversial, there is increasing evidence that the assembly of both the LEs and the CE is a critical step for the correct progression and outcome of meiosis in most organisms (von Wettstein et al. 1984; Page and Hawley 2004; Heyting 2005). However, the presence and also the dynamics of assembly and disassembly of the SC seem to vary among eukaryotes. In Schyzosaccaromyces pombe, a tripartite SC is absent between homologous chromosomes (Olson et al. 1978). A similar situation is found during Drosophila melanogaster male meiosis (Rasmussen 1973). On the other hand, in Bombyx mori females, whose homologous chromosomes do not recombine, the $\mathrm{SC}$ is not disassembled at diplotene, but it is maintained until metaphase-I (Rasmussen 1977). These divergencies clearly illustrate that the relevance of $\mathrm{SC}$ assembly is not equal for all species. Moreover, in some cases, the dynamics of SC assembly differs even between chromosomes of the same complement. Sex chromosomes represent a classical example of this situation.

Sex chromosomes in species with XY or ZW determining systems are genetically and morphologically differentiated. There are species, many fishes for instance, in which this differentiation only involves a small region of the sex chromosomes (Solari 1993). Conversely, sex chromosomes in almost all eutherian mammals only preserve a small region of homology. The extreme situation is found in some eutherian and in almost all marsupial mammals, in which sex chromosomes do not share any homologous region (Solari and Ashley 1977; Wolf et al. 1988; Carnero et al. 1991; Hayman 1990; Graves 1996). Moreover, in the species with $\mathrm{XX} / \mathrm{X} 0$ sex determining system, many insects for instance, the heterogametic sex only bears a copy of the X chromosome.

The genetic and morphological differentiation of sex chromosomes has important consequences for their meiotic
Fig. 1 Relative distribution of SYCP3 and cohesin subunits during pachytene in mouse spermatocytes. The axial elements of sex chromosomes $(\mathrm{X}, \mathrm{Y})$ appear thickened, and show characteristic splittings (arrows) and excrescences (arrowheads). In contrast, the outline of the autosomal SC (A) is regular. The PAR is indicated by an asterisk (*). The pattern of SYCP3 distribution is compared to those of SYCP1 $(\mathbf{a}-\mathbf{c}), \mathrm{SMC} 3$ (d-f), STAG3 (g-i), REC8 $(\mathbf{j}-\mathbf{l})$, and RAD21 (m-o). Note the presence of SYCP1 in the unsynapsed sex AEs. Most cohesins follow the modifications of the AEs in the sex chromosomes, excepting REC8 that is mostly concentrated in the synapsed region but is less abundant in the unsynapsed ones

\section{Pachytene}
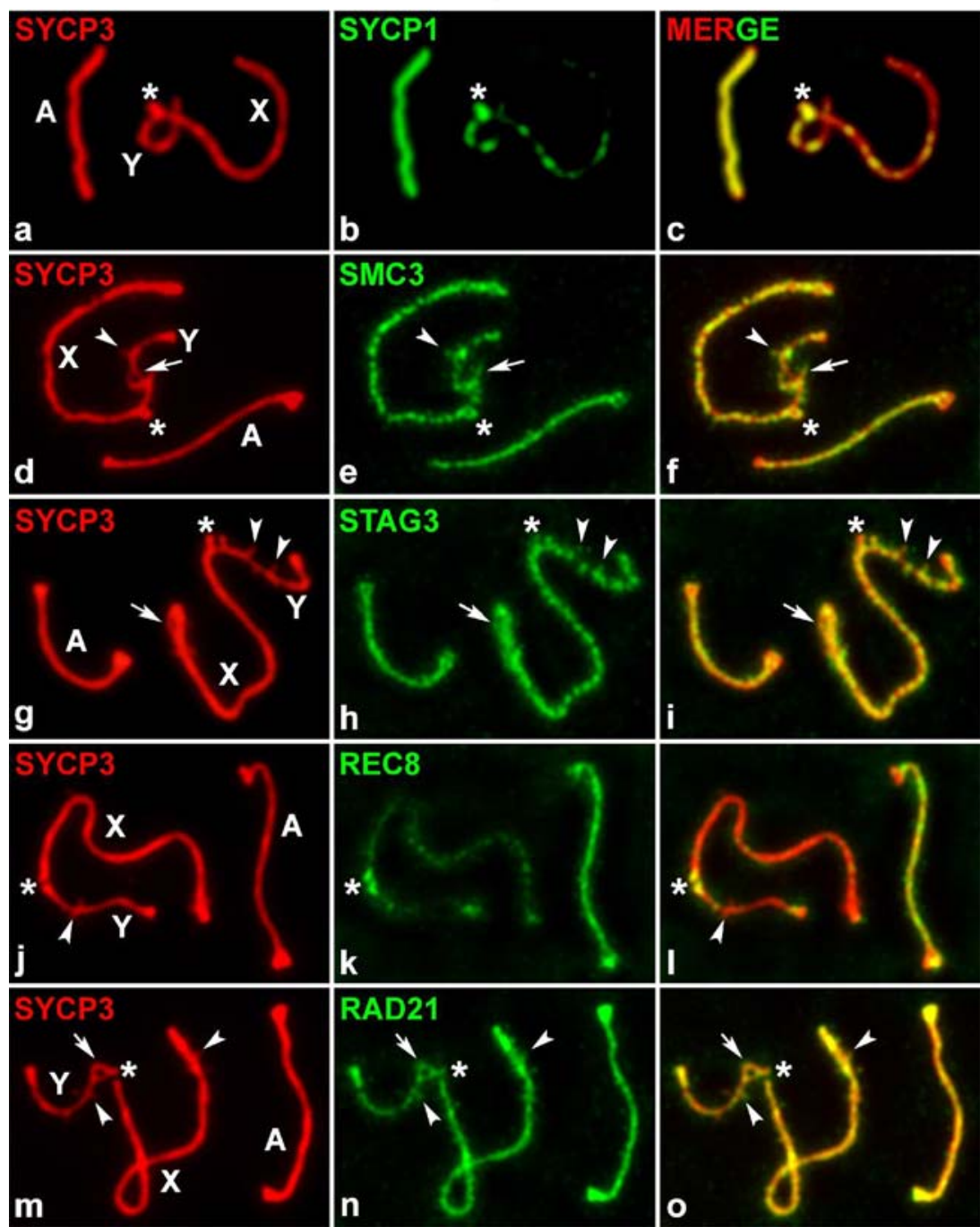
behavior. The most obvious is that their nonhomologous regions usually appear unsynapsed during the first meiotic prophase. This is frequently accompanied by conspicuous modifications in the AEs of sex chromosomes. In this paper, we reexamine some of the modifications of the structure and dynamics of the SC on the sex chromosomes of diverse groups of animals, and compare them with the behavior of the subjacent cohesin axis. The analysis involves sex chromosomes of eutherian and marsupial mammals, orthopterans, and hemipterans, whose pattern of sex chromosome behavior during meiosis has been a classical matter of study. We illustrate the most outstanding features with our own observations, some of which have not been previously published. The comparison between these animal groups reveals the different strategies adopted by species that have to deal with partially or completely asynaptic sex chromosomes. It is interesting to note that differences between the organization of the SC and the cohesin axis in sex chromosomes are also observed.

\section{Sex chromosomal AEs are highly modified in eutherian mammals}

The existence of a region of synapsis between sex chromosomes in mammals, firstly proposed by Koller and Darlington (1934), generated a dispute that lasted more than 30 years, until it was definitively demonstrated by Solari 1969, 1970a,b (reviewed in Solari 1974). These and further studies also revealed that the unsynapsed AEs of sex chromosomes in eutherians present a number of structural modifications that are particularly conspicuous during late pachytene and diplotene: (1) AEs appear thickened; (2) they often split into several threads; (3) they present an irregular outline; and (4) they show excrescences emanating in some places along their length (Solari 1970a,b; Tres 1977; Goetz et al. 1984). It is interesting to note that these features seem to be exclusive of the unsynapsed regions of sex chromosomes because the LEs involved in the formation of the short segment of tripartite $\mathrm{SC}$ in the pseudoautosomal region (PAR) are similar to the LEs of autosomes. In fact, LEs also show normal morphology in the region of synapsis between the sex chromosomes that during early pachytene extends beyond the PAR (Solari 1970a; Tres 1977; Goetz et al. 1984). Thus, the modification of the sex chromosomal AEs seems to be a consequence of their asynaptic nature.

Immunolabeling of SC proteins has indicated that most of the structural modifications shown by the unsynapsed AEs of sex chromosomes correlate with a differential deposition of SYCP2 and SYCP3 proteins, that are the main components of the AEs/LEs in mammals (Lammers et al. 1994; Dobson et al. 1994; Moens and Spyropoulos 1995; Offenberg et al. 1998). Moreover, SYCP1, the main component of TFs and the CE (Meuwissen et al. 1992), may also contribute to these modifications. This protein, considered to be exclusive of synapsed regions, has also been found to be associated to unsynapsed autosomal AEs (Heyting et al. 1987). The detailed analysis of SYCP1 distribution reveals that it also appears associated to the single AEs of both $\mathrm{X}$ and $\mathrm{Y}$ chromosomes (Fig. 1a-c). Although it is not known which factors promote the association of SYCP1 with these unsynapsed regions, it is likely that, in the thickened AEs of sex chromosomes, SYCP2 and SYCP3 form a structural framework for the deposition of SYCP1. This could be the case of the regions where AEs split into several filaments, a feature described for both sex and autosomal chromosomes (Solari 1974; del Mazo and Gil-Alberdi 1986; Dietrich et al. 1992). Thus, sex chromosomal AEs show particular structure and peculiar protein composition.

\section{The organization and composition of the cohesin axis are also modified on the eutherian sex chromosomes}

The structural modifications of the sex chromosomes may not be exclusively due to SC components. In the last years, an increasing number of proteins have been shown to be either associated or adjacent to the AEs. The first group corresponds to the cohesin complex that includes: SMC1 $\alpha$ (Eijpe et al. 2000), SMC1 $\beta$ (Revenkova et al. 2001), SMC3 (Eijpe et al. 2000), REC8 (Eijpe et al. 2003; Lee et al. 2003), STAG3 (Prieto et al. 2001), and RAD21 (Parra et al. 2004; Xu et al. 2004). Three of these proteins, namely SMC1 $\beta$, STAG3, and REC8, are exclusively synthesized during meiosis, whereas $\mathrm{SMC} 1 \alpha, \mathrm{SMC} 3$, and $\mathrm{RAD} 21$ are present in both somatic and meiotic cells. The components of the cohesin complex are thought to form a cohesin axis subjacent to the AEs. Moreover, the AEs and the cohesin axes are independent structures, as revealed by the presence of the latter in SYCP3 defective mouse (Pelttari et al. 2001). However, the analysis of the distribution of cohesin complexes during pachytene reveals that many of the structural modifications of sex chromosomal AEs during this stage, such as the splittings and the excrescences, are also encompassed by some of the cohesin subunits, namely SMC3, STAG3, and RAD21 (Fig. 1) and also probably SMC1 $\beta$ (Revenkova et al. 2001). It is interesting to note that REC 8 behavior is distinct because this protein seems to be less abundant in the sex than in the autosome AEs, excepting in the PAR, where it is markedly accumulated at the end of both sex chromosomes (Fig. 1j-1) (Eijpe et al. 2003; Lee et al. 2003). REC8 has been described to be a cohesin subunit exclusively present in meiosis, and it probably replaces RAD21 in the cohesin complex (Eijpe et al. 2003). The preferential location of REC8 in both autosomes and the synapsed regions of the sex chromosomes may indicate that this subunit is involved in the formation of cohesin complexes that are specific of those regions with a tripartite SC. Whether this is either a consequence or a primary cause of synapsis is an interesting question for further studies.

It is noticeable that the pattern of location of the components of the AEs and the cohesin subunits in the sex chromosomes is different during diplotene. At this stage, the sex AEs show prominent and irregular thickenings that are detectable by SYCP3 labeling. Most cohesins, SMC3, 
STAG3, and remarkably REC8, do not follow this pattern of distribution and show almost identical locations along the sex chromosomes (Fig. 2). However, RAD21, another cohesin subunit, follows an identical distribution to SYCP3 and encompasses the modifications of the AEs (Fig. 2j-1). This is in agreement with the previous reports indicating that RAD21 colocalizes throughout meiosis with SYCP3 (Parra et al. 2004).

Three main conclusions can be drawn from this analysis. First, the modifications in sex chromosomal AEs during pachytene are accompanied by similar changes of the subjacent cohesin axis. These peculiarities may be mainly due to the unsynaptic nature of these AEs, although autosomal AEs/LEs do not show such modifications when they appear as single structures during zygotene or diplotene.

Second, the organization of sex chromosomes changes at the pachytene/diplotene transition: the morphology of the AEs changes and the morphology and composition of the cohesin axis are also modified. However, these changes are not the same in AEs and cohesin axes. This indicates that they represent different structures, and must be regulated in different ways during some stages of the first meiotic prophase. Significantly, most cohesins do not follow the modifications of the LEs in oocytes (Prieto et al. 2004). Thus, this feature is not exclusive of neither sex chromosomes nor male meiosis.

Third, the differential pattern of distribution of some cohesion subunits indicates the presence of distinct cohesin complexes assembled on the sex chromosomes, which can differ in their structural and/or functional roles. This is the case of REC8 and RAD21. As mentioned, REC 8 is a meiosis-specific cohesin subunit, which has been proposed to replace RAD21 during this cell division. However, RAD21 is actually expressed during meiosis (Prieto et al. 2002) and moreover, it seems to interact with the SYCP2 and SYCP3 components of the AEs (Parra et al. 2004). Consequently, besides its role in sister chromatid cohesion during meiosis, RAD21 may play additional roles in the structural organization of sex chromosomes, and also of the autosomes, that are different from those played by REC8.

\section{BRCA1 and ATR associate specifically to unsynapsed sex chromosomal AEs in mammals}

In addition to $\mathrm{SC}$ and cohesin complex proteins, present in both autosomes and sex chromosomes, another group of components that may influence the structure of sex chromosomes is constituted by proteins that specifically associate to sex chromosome AEs. The list includes: XY40 (Smith and Benavente 1992; Alsheimer et al. 1997), BRCA1 (Scully et al. 1997), ATR (Keegan et al. 1996), XMR (Escalier and Garchon 2000), and also Xist RNA, a nontranslated RNA involved in the inactivation of the $\mathrm{X}$ chromosomes in female somatic cells and $\mathrm{X}$ and $\mathrm{Y}$ chromosomes in male meiotic cells (Ayoub et al. 1997). While the function of many of these proteins remains
Fig. 2 Relative distribution of SYCP3 and cohesin subunits during diplotene in mouse spermatocytes. The axial elements of sex chromosomes (X, Y) appear thickened, and show an irregular outline (arrowheads). The outline of the desynapsed autosomal LEs (A) is regular. The PAR is indicated by an asterisk $(*)$. The pattern of SYCP3 distribution is compared to that of with SMC3 $(\mathbf{a}-\mathbf{c})$, STAG3 (d-f), REC8 (g-i), and RAD21 (j-l) (green). Note that all cohesins except RAD21 depart from the morphological differentiations of the sex chromosomal AEs

\section{Diplotene}

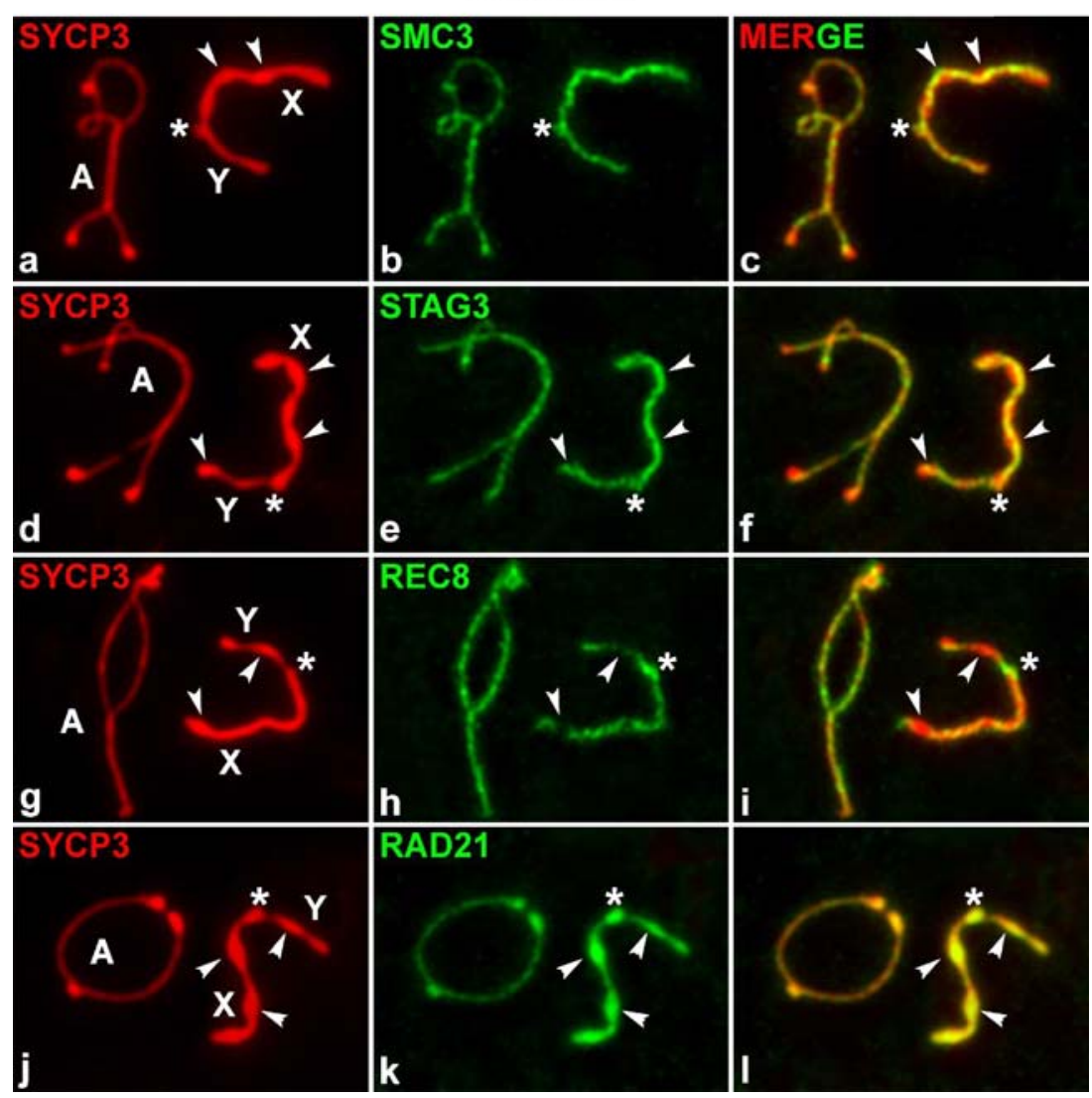


uncertain, recent works have yielded important clues as regards the roles of BRCA1 and ATR. Both proteins localize to the unsynapsed AEs in both autosomes and sex chromosomes during zygotene. However, during pachytene, their distribution is restricted to the unsynapsed regions of the sex chromosomes (Keegan et al. 1996; Scully et al. 1997; Moens et al. 1999). It has been proposed that BRCA1 and ATR are targeted to unsynapsed AEs at late zygotene, and they are involved in triggering the process of meiotic sex chromosome inactivation (MSCI) (Turner et al. 2004). Furthermore, ATR, a phosphoinositide-3-kinase-related kinase, also localizes in the chromatin of both sex chromosomes, where it could phosphorylate the histone variant $\mathrm{H} 2 \mathrm{AX}$, a necessary step for the initiation of MSCI (Fernandez-Capetillo et al. 2003; Turner et al. 2004; Bellani et al. 2005). The immunolocalization of these two proteins reveals that ATR encompasses the modifications of AEs during pachytene and early diplotene, whereas BRCA1 does not (Fig. 3). Furthermore, BRCA1 disappears from the sex chromosomes at the beginning of diplotene, while ATR remains associated to them until late diplotene (Fig. 3g-1). The differential pattern of location of both proteins indicates that although they may be functionally linked, ATR performs additional functions through its interaction with other components of the axial structures of the sex chromosomes.

\section{The overcrowded axes of eutherian sex chromosomes and sex chromatin conformation}

All the previous data indicate that in eutherian sex chromosomes, axial structures are a complex in which a wide range of proteins is assembled. It is interesting to note that many of these components also appear distributed in the autosomes, although they are not modified in the same way as they are in the sex chromosomes. It is difficult to ascertain which factors could be responsible of this special behavior. It is likely that the unsynapsed condition of sex chromosomes and the specific association of some components to these regions could have an important role. BRCA1, ATR, and $\gamma \mathrm{H} 2 \mathrm{AX}$, the phosphorylated form of H2AX, could be some of these components. However, these proteins also appear localized in the unsynapsed autosomal regions of mouse bearing chromosomal trans-
Fig. 3 Relative distribution of SYCP3, BRCA1, and ATR during pachytene and diplotene in mouse spermatocytes. Both BRCA1 and ATR only localize in the unsynapsed AEs and are absent from the PAR (asterisk). BRCA1 clearly does not appear in the excrescences (arrowheads) or thickenings (arrows) of the sex AEs and completely disappears at the beginning of diplotene. ATR follows a pattern of localization similar to that of SYCP3 during pachytene and diplotene, although disappears at late diplotene. This protein also localizes in the chromatin of sex chromosomes during pachytene. Both BRCA1 and ATR are completely absent in the autosomes (A)

\section{Pachytene}
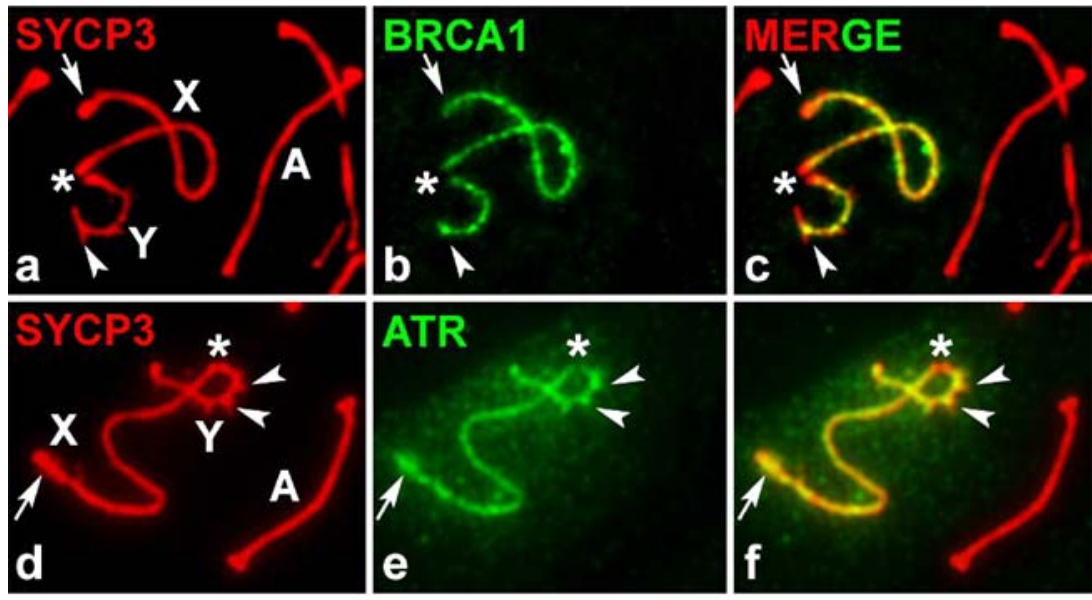

\section{Diplotene}
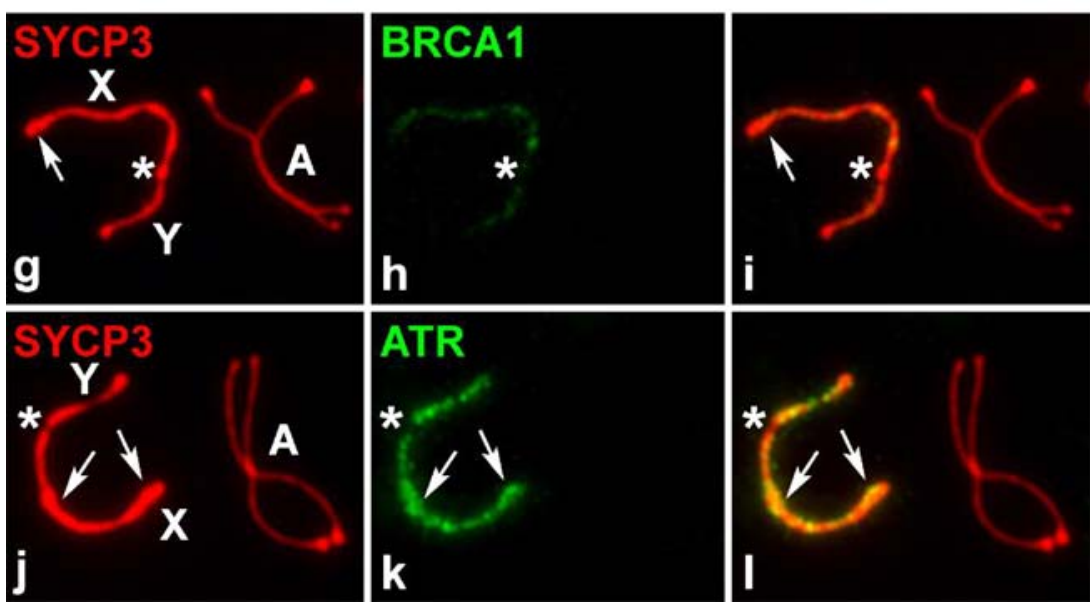
locations (Turner et al. 2005), even though these regions do not suffer morphological modifications in their AEs.

Therefore, besides the asynaptic nature of the AEs of sex chromosomes, other factors may be acting to induce their modification. In this sense, it is important to take into account the special behavior of sex chromosomes as regards their chromatin condensation and inactivation, which gives rise to sex chromosomes as a compacted chromatin domain, i.e., the sex body (Solari 1974; McKee and Handel 1993). Perhaps some of the many proteins that associate to the sex chromosomes during the first meiotic prophase (review by Hoyer-Fender 2003; Handel 2004) may induce a specific and differential conformation of the sex chromatin, which could have an influence on the axial organization of these chromosomes. Nevertheless, one should note that inactivation would not be sufficient to originate these changes because autosomal unsynapsed regions, which incorporate BRCA1, ATR, and $\gamma \mathrm{H} 2 \mathrm{AX}$, are also inactivated (Turner et al. 2005), but do not suffer such modifications. Hence, one can speculate that other conditions, previous or subsequent to the initiation of MSCI, are necessary to remodel sex chromosome organization.

\section{Marsupial sex chromosomal AEs expand at their ends to form a pairing structure}

An extreme case of modification in the AEs of sex chromosomes has been described in marsupial males. In most marsupial species, sex chromosomes do not possess a PAR and sex chromosomes do not synapse at all. Instead, they develop a specific structure, called dense plate, which maintains their association (Solari and Bianchi 1975; Sharp 1982; Roche et al. 1986; Seluja et al. 1987). This structure is formed by the modification of sex chromosomal AEs, which expand on the nuclear envelope giving rise to a dishlike structure in which the ends of the $\mathrm{X}$ and Y AEs are immersed (Fig. 4a,b). According to this, the dense plate is mainly formed by the SYCP3 component of the AEs (Fig. 4c,d) (Page et al. 2003). It is interesting to note that although the cohesin subunits SMC3 and STAG3 are present along sex chromosomes, they do not follow many of the modifications of the sex chromosomal AEs and, most relevantly, do not participate in the organization of the dense plate (Page et al. 2005).

The modification of the AEs that originates the dense plate in marsupial males is indicative that the structural elements of the SC may play, in addition to the organization of the canonical LEs, other roles in nonhomologous chromosomes pairing (Page et al. 2003) and segregation (Page et al. unpublished). These additional roles of the SC elements are not exclusive of marsupial meiosis. In many invertebrate species, the SC may also play important roles in the segregation of nonexchanged chromosomes (reviewed by Wolf 1994).

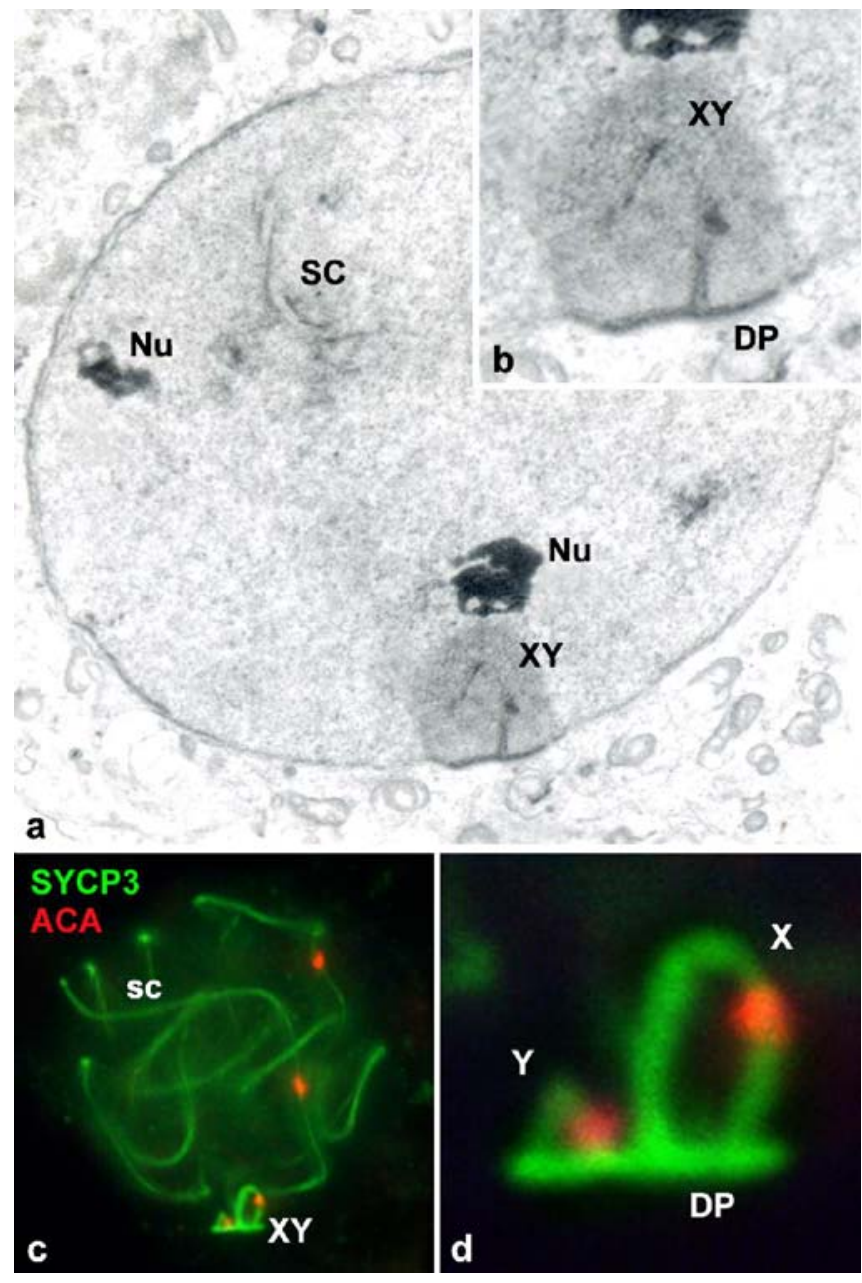

Fig. 4 The marsupial dense plate. a Image of the nucleus of a Dromiciops gliroides spermatocyte in pachytene processed with conventional techniques for electron microscopy. An autosomal SC (SC) and the nucleoli $(\mathrm{Nu})$ are visible. The sex body $(\mathrm{XY})$ appears at the periphery of the nucleus. $\mathbf{b}$ At a higher magnification the axis of the X chromosome and the dense plate (DP) are clearly visible. c-d Immunolocalization of SYCP3 (green) and centromeres (ACA) (red) in a pachytene spermatocyte of the marsupial Dromiciops gliroides. The AEs of the sex chromosomes (X, Y) appear thickened but present a regular outline. The dense plate (DP) is seen as a structure strongly labeled with SYCP3 associated to the ends of sex chromosomal AEs

\section{Sex chromosomes in orthopterans do not show any structural modification}

Although most of the studies on sex chromosome structure and behavior have been focused in mammals, the study of other groups of organisms brings valuable information for understanding the peculiar biology of these chromosomes. Orthopterans are among the most used species in classical meiotic studies because of their low chromosome number and their large-sized chromosomes. Most grasshopper species present an $\mathrm{XX} / \mathrm{X} 0$ sex determining mechanism; hence, during male meiosis the single $\mathrm{X}$ chromosome behaves as a univalent. The ultrastructure of the $\mathrm{X}$ chromosome in grasshoppers has been analyzed in both sections and surface spreads (Moens 1969; Counce and 
Meyer 1973; Solari and Counce 1977) revealing that this chromosome forms a single thin AE that, contrary to what is detected in the unsynapsed sex chromosome segments of mammals, has a normal appearance (Fig. 5a). Moreover, this regular organization is also maintained in species with neo XY males (JL Santos unpublished).

The identification of SC proteins in these species has been precluded by the absence of labeling by antibodies raised against mammalian or Drosophila SC proteins (Moens et al. 1987), but some components of the cohesin complex have been analyzed in Locusta migratoria, Eyprepocnemys plorans, and Stethophyma grossum (Viera et al. 2004a,b; Calvente et al. 2005). SMC3 labeling

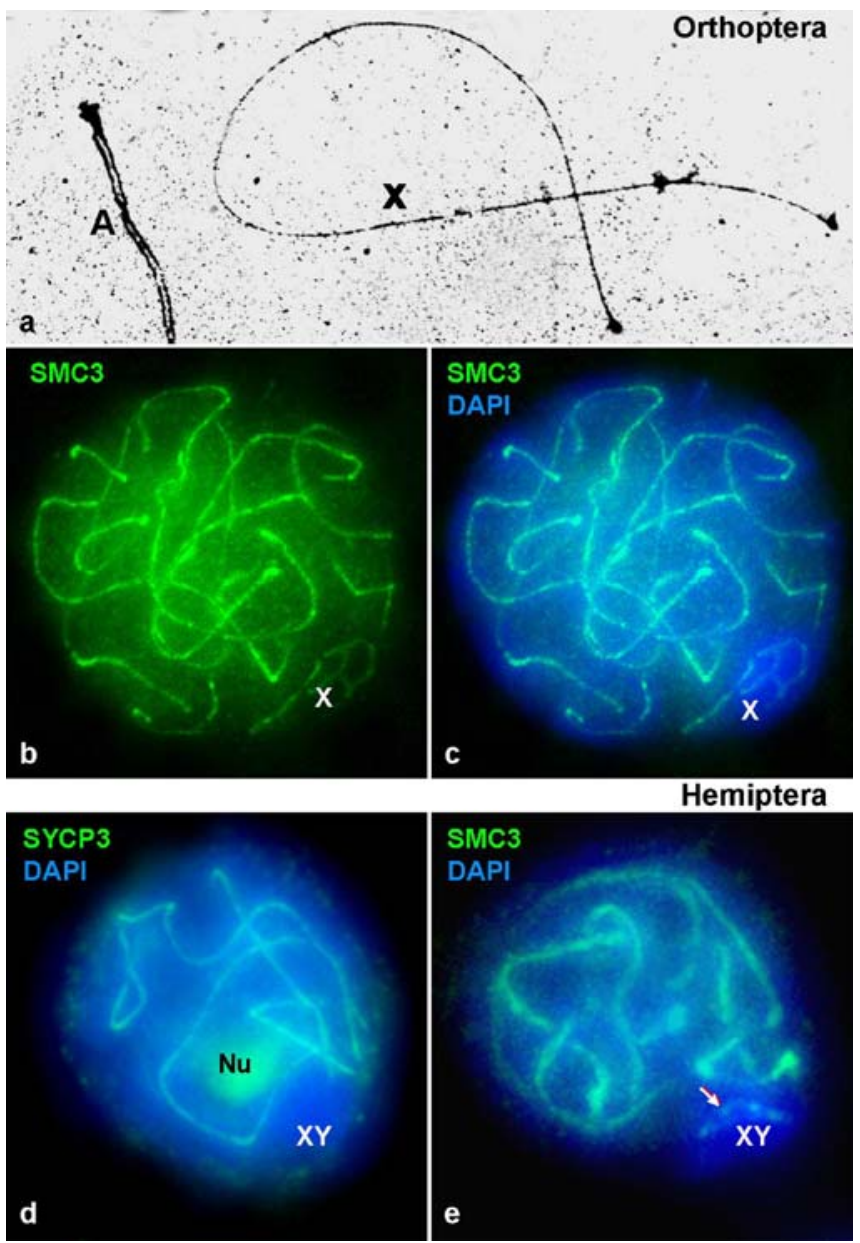

Fig. 5 Axial elements and cohesin axes in insect sex chromosomes. a Electron microscopy image of the single $\mathrm{AE}$ of the $\mathrm{X}$ chromosome of a grasshopper obtained by silver staining of a surface spread spermatocyte. This AE is similar to the LEs of the autosomal bivalents (A) and does not show any structural modification. $\mathbf{b}-\mathbf{c}$ Immunolocalization of SMC3 in a spermatocyte of Locusta migratoria. The $\mathrm{X}$ chromosome $(\mathrm{X})$ presents an axial structure labeled by SMC3. d Immunolocalization of SYCP3 in a spermatocyte of the hemipteran Graphosoma italicum. This protein is present in the autosomes but does not localize in the sex chromosomes (XY). Note the SYCP3 labeling in the nucleolus $(\mathrm{Nu})$. e Immunolocalization of SMC3 in a spermatocyte of the same species. In this case, an axial structure (arrow) is detected in the sex chromosomes (XY). The three-dimensional reconstruction movies of the nuclei shown in $\mathbf{b}-\mathbf{e}$ are included as electronic supplementary material and can be seen at Chromosoma web page indicates the formation of a thin cohesin axis on the $\mathrm{X}$ chromosome (Fig. 5b,c). Thus, the structure of the $\mathrm{X}$ chromosome seems to be unaltered by its unsynapsed condition. Similar results have been obtained in B chromosomes present as univalents in some grasshoppers (Viera et al. 2004a).

In grasshoppers, SC disassembles at the beginning of diplotene, and both LEs and the CE are disorganized (Solari and Counce 1977; Rufas et al. 1992; Molina et al. 1998). Likewise, the organization of the cohesin axes in autosomes is highly modified during the transition pachytene/diplotene. It is interesting to note that theses changes do not seem to affect the sex chromosome because its cohesin axis preserves a very similar organization throughout these stages (Viera et al. 2004a,b).

Sex chromosome behavior in male grasshoppers illustrates some interesting features: (1) the organization of its $\mathrm{AE}$ remains unaltered throughout the first meiotic prophase. Because the $\mathrm{X}$ chromosome in orthopterans, as in mammals, is inactive and remains condensed throughout first meiotic prophase, there is no direct relationship between condensation and AE modification; (2) the organization of the $\mathrm{X}$ chromosome changes at the beginning of diplotene; and (3) although the X chromosome $\mathrm{AE}$ and the subjacent cohesin axis show a similar behavior during pachytene, they show striking differences at the onset of diplotene. As we have previously indicated, these two last features are also present in mammals.

\section{Hemipterans lack AEs on their sex chromosomes but they have a cohesin axis}

A completely different situation is found in hemipteran insects. The species of this Order present a series of striking cytogenetic features. First, they possess holocentric chromosomes. In mitotic divisions, the kinetochore occupies almost the entire length of the chromosomes (HughesSchrader and Schrader 1961). However, these chromosomes behave as monocentric during meiosis (Schrader 1939), and instead of organizing a kinetochore structure, the microtubules directly enter into the chromatin (Rufas and Giménez-Martín 1986). Second, sex chromosomes neither synapse nor recombine and they appear as univalents during metaphase-I. But instead of segregating during the first meiotic division, both sex chromosomes separate their chromatids at anaphase-I, undergoing an equational division. The reductional segregation of sex chromatids occurs during the second meiotic division. This process in which the common sequence of segregation is altered is called inverted meiosis (Ueshima 1979; Gonzalez-Garcia et al. 1996).

Besides these unusual features, sex chromosomes in all hemipteran species studied to date do not form an $\mathrm{AE}$ during the first meiotic prophase. This conclusion emerged from ultrastructural studies, which have failed to detect AEs along sex chromosomes neither in spreads nor in sections (Solari 1979; Suja et al. 2000; Pigozzi and Solari 2003), although a fuzzy core of undetermined nature is 
detected in the sex chromosomes of Triatoma infestans and T. pallidipennis (Pigozzi and Solari 2003). Furthermore, SYCP3, the main component of the LEs in mammals, is present during pachytene in the autosomes, but is absent from the sex chromosomes (Fig. 5d). This feature is independent of the mechanism of sex chromosome determination because it is common to species with $\mathrm{XY}, \mathrm{X} 0$, $\mathrm{X}_{1} \mathrm{X}_{2} \mathrm{Y}$, or $\mathrm{X}_{1} \mathrm{X}_{2} 00$ males (Suja et al. 2000).

The absence of AEs in hemipteran sex chromosomes seems to be accompanied by the absence of meiotic cohesins in these chromosomes. It has been reported that REC8 localizes along the autosomes in Triatoma species, but is absent in the sex chromosomes (Pigozzi and Solari 2003). This feature may explain the equational segregation of sister chromatids of both sex chromosomes during the first meiotic division. However, we have found that SMC3, another component of the cohesin complex, does localize in the autosomes and also in the sex chromosomes of Graphosoma italicum (Fig. 5e). SMC3 appears as linear elements in the $\mathrm{X}$ and $\mathrm{Y}$ chromosomes, indicating the formation of a cohesin axis. This axis may correspond to the fuzzy cores detected in the sex chromosomes of Triatoma infestans and T. pallidipennis (Pigozzi and Solari 2003). Therefore, it seems that, as occurs in mammals, several cohesin complexes, which differ in composition and location, are assembled in the meiotic chromosomes of hemipterans. Moreover, the meiosis-specific cohesin REC8 presents a particular distribution, and is absent in the sex chromosomes. In any case, the absence of AEs in the sex chromosomes of hemipterans remains a mystery. Obviously, it cannot be related to the absence of a subjacent cohesin axis. Consequently, other structural or regulatory mechanisms must explain this special behavior.

\section{Concluding remarks}

Sex chromosomes have been found in almost all groups of animals, some plants and also in fungi (Fraser and Heitman 2005). Classical studies in vertebrates and flies indicated that one of the fundamental events leading to the differentiation of sex chromosomes is the regional suppression of recombination around the loci involved in sex determination, that is, the origin of sex chromosomes is at the very heart of meiosis (Ohno 1967; Charlesworth 1991; Rice 1996). Further steps of differentiation involve the reiterated occurrence of chromosomal rearrangements (Lahn and Page 1999). It is interesting to note that the mechanisms that drive sex chromosome evolution seem to be common to all eukaryotes, and therefore it is likely that some of the features described for animals are also applicable to plants and fungi (Charlesworth 2002; Fraser and Heitman 2005). In any case, these processes ultimately lead to the morphological differentiation of sex chromosomes. As we have seen, this has fundamental consequences for the behavior of these chromosomes during meiosis. However, while the genetic mechanisms behind sex chromosome evolution may be common in different organisms, the structural modifications undergone by these chromosomes during meiosis greatly vary in different groups. This could be a consequence of the different origins of sex chromosomes, but could also be due to the existence of different strategies adopted by different organisms during evolution. In this sense, the SC in these chromosomes shows significant variations, ranging from the conspicuous modifications found in mammals to the absence of AEs in hemipterans. On the other hand, the cohesin axes are more stable. They are always present in sex chromosomes, albeit some of the components, namely REC8, show a peculiar behavior, rather different from the other cohesin subunits.

Acknowledgements We are indebt to Dr. Carlos García de la Vega for his helpful comments on the manuscript, and to Dr. Christa Heyting (Wageningen, The Netherlands) and Dr. José Luis Barbero (Madrid, Spain) for providing antibodies. This work was supported by grants BMC2002-00043 and BFU2005-05668-C03-01 from Ministerio de Educación y Ciencia (Spain), grant 1001160016 from Universidad Autónoma de Madrid (Spain), FONDECYT grants 2000008, 1040910, and 7040174 (Chile), and a grant from Centro de Estudios de América Latina-BSCH (Spain). RdlF received support from FGUAM and Olympus Optical España S.A.

\section{References}

Alsheimer M, Imamichi Y, Heid H, Benavente R (1997) Molecular characterization and expression pattern of $\mathrm{XY}$ body-associated protein XY40 of the rat. Chromosoma 106:308-314

Ayoub N, Richler C, Wahrman J (1997) Xist RNA is associated with the transcriptionally inactive $\mathrm{XY}$ body in mammal male meiosis. Chromosoma 106:1-10

Bellani MA, Romanienko PJ, Cairatti DA, Camerini-Otero RD (2005) SPO11 is required for sex-body formation, and Spo11 heterozygosity rescues the prophase arrest of $\mathrm{Atm}^{-1-}$ spermatocytes. J Cell Sci 118:3233-3245

Calvente A, Viera A, Page J, Parra MT, Gomez R, Suja JA, Rufas JS, Santos JL (2005) DNA double-strand breaks and homology search: inferences from a species with incomplete pairing and synapsis. J Cell Sci 118:2957-2963

Carnero A, Jimenez R, Burgos M, Sanchez A, Diaz de la Guardia R (1991) Achiasmatic sex chromosomes in Pitymys duodecimcostatus: mechanisms of association and segregation. Cytogenet Cell Genet 56:78-81

Charlesworth B (1991) The evolution of sex chromosomes. Science 251:1030-1033

Charlesworth D (2002) Plant sex determination and sex chromosomes. Heredity 88:94-101

Counce SJ, Meyer GF (1973) Differentiation of the synaptonemal complex and the kinetochore in Locusta spermatocytes studied by whole mount electron microscopy. Chromosoma 44:231-253

del Mazo J, Gil-Alberdi L (1986) Multistranded organization of the lateral elements of the synaptonemal complex in the rat and mouse. Cytogenet Cell Genet 41:219-224

Dietrich AJ, van Marle J, Heyting C, Vink AC (1992) Ultrastructural evidence for a triple structure of the lateral element of the synaptonemal complex. J Struct Biol 109:196-200

Dobson MJ, Pearlman RE, Karaiskakis A, Spyropoulos B, Moens PB (1994) Synaptonemal complex proteins: occurrence, epitope mapping and chromosome disjunction. J Cell Sci 107: 2749-2760

Earnshaw WC, Halligan B, Cooke CA, Heck MM, Liu LF (1985) Topoisomerase II is a structural component of mitotic chromosome scaffolds. J Cell Biol 100:1706-1715

Eijpe M, Heyting, C, Gross B, Jessberger R (2000) Association of mammalian SMC1 and SMC3 proteins with meiotic chromosomes and synaptonemal complex. J Cell Sci 113:673-682 
Eijpe M, Offenberg H, Jessberger R, Revenkova E, Heyting C (2003) Meiotic cohesin REC8 marks the axial elements of rat synaptonemal complexes before cohesins SMC1beta and SMC3. J Cell Biol 160:657-670

Escalier D, Garchon HJ (2000) XMR is associated with the asynapsed segments of sex chromosomes in the XY body of mouse primary spermatocytes. Chromosoma 109:259-265

Fawcett DW (1956) The fine structure of chromosomes in the meiocyte prophase of vertebrate spermatocyte. J Biophys Biochem Cytol 2:403-406

Fernandez-Capetillo O, Mahadevaiah SK, Celeste A, Romanienko PJ, Camerini-Otero RD, Bonner WM, Manova K, Burgoyne P, Nussenzweig A (2003) H2AX is required for chromatin remodeling and inactivation of sex chromosomes in male mouse meiosis. Dev Cell 4:497-508

Fraser JA, Heitman J (2005) Chromosomal sex-determining regions in animals, plants and fungi. Curr Opin Genet Dev 15:645-651

Goetz P, Chandley AC, Speed RM (1984) Morphological and temporal sequence of meiotic prophase development at puberty in the male mouse. J Cell Sci 65:249-263

Gonzalez-Garcia JM, Antonio C, Suja JA, Rufas JS (1986) Meiosis in holocentric chromosomes: kinetic activity is randomly restricted to the chromatid ends of sex univalents in Graphosoma italicum (Heteroptera). Chromosome Res 4: 124-132

Graves JAM (1996) Mammals that break the rules: genetics of marsupials and monotremes. Annu Rev Genet 30:233-260

Handel MA (2004) The XY body: a specialized meiotic chromatin domain. Exp Cell Res 296:57-63

Hayman DL (1990) Marsupial cytogenetics. Aust J Zool 37:331-349

Heyting C (2005) Meiotic transverse filament proteins: essential for crossing over. Transgenic Res 14:547-550

Heyting C, Moens PB, van Raamsdonk W, Dietrich AJ, Vink AC, Redeker EJ (1987) Identification of two major components of the lateral elements of synaptonemal complexes of the rat. Eur J Cell Biol 43:148-154

Hoyer-Fender S (2003) Molecular aspects of XY body formation. Cytogenet Genome Res 103:245-255

Hughes-Schrader S, Schrader F (1961) The kinetochore of the Hemiptera. Chromosoma 12:327-350

Keegan KS, Holtzman DA, Plug AW, Christenson ER, Brainerd EE, Flaggs G, Bentley NJ, Taylor EM, Meyn MS, Moss SB, Carr AM, Ashley T, Hoekstra MF (1996) The Atr and Atm protein kinases associate with different sites along meiotically pairing chromosomes. Genes Dev 10:2423-2437

Koller PC, Darlington CD (1934) The genetical and mechanical properties of the sex chromosomes. I. Rattus norvergicus, $\mathrm{O}$. J Genet 29:159-173

Lahn BT, Page DC (1999) Four evolutionary strata on the human X chromosome. Science 286:964-967

Lammers JHM, Offenberg HH, van Aalderen M, Vink ACG, Dietrich AJJ, Heyting C (1994) The gene encoding a major component of the lateral elements of synaptonemal complexes of the rat is related to X-linked lymphocyte-regulated genes. Mol Cell Biol 14:1137-1146

Lee J, Iwai T, Yokota T, Yamashita M (2003) Temporally and spatially selective loss of Rec8 protein from meiotic chromosomes during mammalian meiosis. J Cell Sci 116:2781-2790

McKee BD, Handel MA (1993) Sex chromosomes, recombination and chromatin conformation. Chromosoma 102:71-80

Meuwissen RLJ, Offenberg HH, Dietrich AJJ, Riesewijk A, van Iersen M, Heyting C (1992) A coiled-coil related protein specific of the synapsed regions of the meiotic prophase chromosomes. EMBO J 11:5091-5100

Moens PB (1969) The fine structure of meiotic chromosome polarization and pairing in Locusta migratoria spermatocytes. Chromosoma 28:1-25

Moens PB, Earnshaw WC (1989) Anti-topoisomerase II recognizes meiotic chromosome cores. Chromosoma 98:317-322

Moens PB, Spyropoulos B (1995) Immunocytology of chiasmata and chromosomal disjunction at mouse meiosis. Chromosoma 104:175-182
Moens PB, Heyting C, Dietrich AJ, van Raamsdonk W, Chen Q (1987) Synaptonemal complex antigen location and conservation. J Cell Biol 105:93-103

Moens PB, Tarsounas M, Morita T, Habu T, Rottinghaus ST, Freire R, Jackson SP, Barlow C, Wynshaw-Boris A (1999) The association of ATR protein with mouse meiotic chromosome cores. Chromosoma 108:95-102

Molina J, Martinez-Flores I, Templado C, Garcia M, Egozcue J (1998) The synaptic process in Locusta migratoria spermatocytes by synaptonemal complex analysis. Histol Histopathol 13:949-954

Moses MJ (1956) Chromosomal structures in crayfish spermatocytes. J Biophys Biochem Cytol 2:215-217

Offenberg HH, Schalk JAC, Meuwissen RLJ, van Aalderen M, Kester HA, Dietrich AJJ, Heyting C (1998) SCP2: a major protein component of the axial elements of synaptonemal complexes of the rat. Nucleic Acids Res 26:2572-2579

Ohno S (1967) Sex chromosomes and sex linked genes. Springer, Berlin Heidelberg New York

Olson LW, Eden U, Egel-Mitani M, Egel R (1978) Asynaptic meiosis in fission yeast? Hereditas 89:189-199

Page J, Berríos S, Rufas JS, Parra MT, Suja JA, Heyting C, Fernandez-Donoso R (2003) The pairing of $X$ and $Y$ chromosomes during meiotic prophase in the marsupial species Thylamys elegans is maintained by a dense plate developed from their axial elements. J Cell Sci 116:551-560

Page J, Berríos S, Parra MT, Viera A, Suja JA, Prieto I, Barbero JL, Rufas JS, Fernandez-Donoso R (2005) The program of sex chromosome pairing in meiosis is highly conserved across marsupial species: implications for sex chromosome evolution. Genetics 170:793-799

Page SL, Hawley RS (2004) The genetics and molecular biology of the synaptonemal complex. Annu Rev Cell Dev Biol 20: $525-558$

Parra MT, Viera A, Gomez R, Page J, Benavente R, Santos JL, Rufas JS, Suja JA (2004) Involvement of the cohesin Rad21 and SCP3 in monopolar attachment of sister kinetochores during mouse meiosis I. J Cell Sci 117:1221-1234

Paulson JR, Laemmli UK (1977) The structure of histone depleted chromosomes. Cell 12:817-828

Pelttari J, Hoja M-R, Yuan L, Liu J-G, Brundell E, Moens P, Santucci-Darmanin S, Jessberger R, Barbero JL, Heyting C, Höög C (2001) A meiotic chromosomal core consisting of cohesin complex proteins recruits DNA recombination proteins and promotes synapsis in the absence of an axial element in mammalian meiotic cells. Mol Cell Biol 21:5667-5677

Pigozzi MI, Solari AJ (2003) Differential immunolocalization of a putative Rec8p in meiotic autosomes and sex chromosomes of triatomine bugs. Chromosoma 112:38-47

Prieto I, Suja JA, Pezzi N, Kremer L, Martinez-A C, Rufas JS, Barbero JL (2001) Mammalian STAG3 is a cohesin specific to sister chromatid arms in meiosis I. Nat Cell Biol 3:761-766

Prieto I, Pezzi N, Buesa JM, Kremer L, Barthelemy I, Carreiro C, Roncal F, Martinez A, Gomez L, Fernandez R, Martinez-A C, Barbero JL (2002) STAG2 and Rad21 mammalian mitotic cohesins are implicated in meiosis. EMBO Rep 3:543-550

Prieto I, Tease C, Pezzi N, Buesa JM, Ortega S, Kremer L, Martinez A, Martinez-A C, Hulten MA, Barbero JL (2004) Cohesin component dynamics during meiotic prophase I in mammalian oocytes. Chromosome Res 12:197-213

Rasmussen SW (1973) Ultrastructural studies of spermatogenesis in Drosophila melanogaster Meigen. Z Zellforsch Mikrosk Anat 140:125-144

Rasmussen SW (1977) The transformation of the Synaptonemal Complex into the 'elimination chromatin' in Bombyx mori oocytes. Chromosoma 60:205-221

Revenkova E, Eijpe M, Heyting C, Gross B, Jessberger R (2001) Novel meiosis-specific isoform of mammalian SMC1. Mol Cell Biol 21:6984-6998

Rice WR (1996) Evolution of the Y sex chromosome in animals. Bioscience 46:331-343 
Roche L, Seluja G, Wettstein R (1986) The meiotic behaviour of the $\mathrm{XY}$ pair in Lutreolina crassicaudata (Marsupialia: Didelphoidea). Genetica 71:213-224

Rufas JS, Giménez-Martín G (1986) Ultraestructure of the kinetochore in Graphosoma italicum (Hemiptera: Heteroptera). Protoplasma 132:142-148

Rufas JS, Santos JL, Diez M, Suja JA (1992) Meiotic chromosome structure: relationship between the synaptonemal complex and the chromatid cores. Genome 35:1054-1061

Saitoh N, Goldberg IG, Wood ER, Earnshaw WC (1994) ScII: an abundant chromosome scaffold protein is a member of a family of putative ATPases with an unusual predicted tertiary structure. J Cell Biol 127:303-318

Schrader F (1939) The structure of the kinetochore at meiosis. Chromosoma 1:230-237

Scully R, Chen J, Plug A, Xiao Y, Weaver D, Feunteun J, Ashley T, Livingston DM (1997) Association of BRCA1 with Rad51 in mitotic and meiotic cells. Cell 88:265-275

Seluja G, Roche L, Solari AJ (1987) Male meiotic prophase in Didelphis albiventris. J Hered 78:218-222

Sharp P (1982) Sex chromosome pairing during male meiosis in marsupials. Chromosoma 86:27-47

Smith A, Benavente R (1992) Meiosis-specific protein selectively associated with sex chromosomes of rat pachytene spermatocytes. Proc Natl Acad Sci USA 89:6938-6942

Solari AJ (1969) The evolution of the ultrastructure of the sex chromosomes (sex vesicle) during the meiotic prophase in mouse spermatocytes. J Ultrastruct Res 27:289-305

Solari AJ (1970a) The spatial relationship of the X and Y chromosomes during meiotic prophase in mouse spermatocytes. Chromosoma 29:217-236

Solari AJ (1970b) The behaviour of chromosomal axes during diplotene in mouse spermatocytes. Chromosoma 31:217-230

Solari AJ (1974) The behavior of the XY pair in mammals. Int Rev Cytol 38:273-317

Solari AJ (1979) Autosomal synaptonemal complexes and sex chromosomes without axes in Triatoma infestans (Reduviidae; Hemiptera). Chromosoma 72:225-240

Solari AJ (1993) Sex chromosomes and sex determination in vertebrates. CRC, Boca Raton

Solari AJ, Bianchi NO (1975) The synaptic behaviour of the X and $\mathrm{Y}$ chromosomes in the marsupial Monodelphis dimidiata. Chromosoma 52:11-25
Solari AJ, Ashley T (1977) Ultrastructure and behavior of the achiasmatic, telosynaptic XY pair of the sand rat (Psammomys obesus). Chromosoma 62:319-336

Solari AJ, Counce SJ (1977) Synaptonemal complex karyotyping in Melanoplus differentialis. J Cell Sci 26:229-250

Suja JA, del Cerro AL, Page J, Rufas JS, Santos JL (2000) Meiotic sister chromatid cohesion in holocentric sex chromosomes of three heteropteran species is maintained in absence of axial elements. Chromosoma 109:35-43

Tres LL (1977) Extensive pairing of the XY bivalent in mouse spermatocytes as visualized by whole-mount electron microscopy. J Cell Sci 25:1-15

Turner JM, Aprelikova O, Xu X, Wang R, Kim S, Chandramouli GV, Barrett JC, Burgoyne PS, Deng CX (2004) BRCA1, histone $\mathrm{H} 2 \mathrm{AX}$ phosphorylation, and male meiotic sex chromosome inactivation. Curr Biol 14:2135-2142

Turner JM, Mahadevaiah SK, Fernandez-Capetillo O, Nussenzweig A, Xu X, Deng CX, Burgoyne PS (2005) Silencing of unsynapsed meiotic chromosomes in the mouse. Nat Genet $37: 41-47$

Ueshima N (1979) Animal cytogenetics. Insecta 6. Hemiptera: Heteroptera. Borntraeger, Berlin

Viera A, Calvente A, Page J, Parra MT, Gomez R, Suja JA, Rufas JS, Santos JL (2004a) X and B chromosomes display similar meiotic characteristics in male grasshoppers. Cytogenet $\mathrm{Ge}-$ nome Res 106:302-308

Viera A, Santos JL, Page J, Parra MT, Calvente A, Cifuentes M, Gomez R, Lira R, Suja JA, Rufas JS (2004b) DNA doublestrand breaks, recombination and synapsis: the timing of meiosis differs in grasshoppers and flies. EMBO Rep 5:385-391

von Wettstein D, Rasmussen SW, Holm PB (1984) The synaptonemal complex in genetic segregation. Annu Rev Genet $18: 331-413$

Wolf KW (1994) How meiotic cells deal with non-exchange chromosomes. Bioessays 16:107-114

Wolf KW, Baumgart K, Winking H (1988) Meiotic association and segregation of the giant sex chromosomes in male field vole (Microtus agrestis). Chromosoma 97:124-133

$\mathrm{Xu} \mathrm{H}$, Beasley M, Verschoor S, Inselman A, Handel MA, McKay MJ (2004) A new role for the mitotic RAD21/SCC1 cohesin in meiotic chromosome cohesion and segregation in the mouse. EMBO Rep 5:378-384 